\title{
Magnetic Medium-Assisted Inverse Bulge Pre- Forming and Deep Drawing Composite Forming Process for Improving Forming Performance of the Cylindrical Parts
}

\author{
Yuan Yuan Mu \\ Harbin University of Science and Technology \\ Feng Li ( $\nabla$ fli@hrbust.edu.cn ) \\ Harbin University of Science and Technology \\ Chao Li \\ Harbin University of Science and Technology \\ Yun Qi Zang \\ Harbin University of Science and Technology \\ Jie Xu \\ Harbin University of Science and Technology
}

\section{Research Article}

Keywords: Magnetorheological (MR) fluid, Inverse bulge pre-forming, Finite element modeling

Posted Date: March 24th, 2021

DOI: https://doi.org/10.21203/rs.3.rs-350410/v1

License: (c) (i) This work is licensed under a Creative Commons Attribution 4.0 International License.

Read Full License 


\section{Abstract}

Due to the poor plasticity of aluminum alloy at room temperature, it is difficult to form thin-walled and complex curved parts. This paper proposes a composite method of inverse bulge pre-forming deep drawing based on intelligent magnetorheological (MR) fluid materials. Through the experiments and finite element modeling of cylindrical parts with drawing ratios of $\mathrm{K}_{1}=2.125$ and $\mathrm{K}_{2}=2.25$ were carried out under different forming conditions. The effect of soft mold medium on the drawing forming of cylindrical parts was studied. The research results show that the uniformity of the wall thickness of the parts is enhanced after using the soft mold medium. When the inverse bulge height is about $9 \mathrm{~mm}$ and $5 \mathrm{~mm}$, the wall thickness variance of the cylindrical part is 0.0023 and 0.0025 pectively, which is reduced by $86.31 \%$ and $82.8 \%$ respectively. In the pre-forming stage, as the height of inverse bulge is increased, the maximum equivalent stress moves from the fillet area of the blank holder to the outer surface of the highest point of the bulging area. Taking drawing ratio of $\mathrm{K}_{1}=2.125$ as an example, the circumferential compressive stress in the flange area decreases and is distributed unifomly under the back pressure and soft drawbeads, the radial stress gradient and equivalent stress gradient at the fillet of die are reduced; For cylindrical parts with drawing ratios of $\mathrm{K}_{1}=2.125$ and $\mathrm{K}_{2}=2.25$, when the inverse bulge height is $9 \mathrm{~mm}$ and $5 \mathrm{~mm}$, the forming effect of the part is the best.

\section{1.introduction}

With the wide application of aluminum alloy materials in the aerospace field, higher requirements are put forward for low density, light weight and performance of materials. Aluminum alloy materials have poor forming performance at room temperature and it is difficult to form thin-walled complex parts [1-3]. Due to the limitations of the forming device and technology in the traditional deep drawing process, the surface of the parts is easy to wrinkle and crack and other defects. Flexible forming technology uses flexible media as forming molds instead of rigid punch or die, such as hydroforming, viscous media forming, and particle forming technology, and because of the difference of forming medium properties, the application fields are different. The use of flexible medium forming technology can not only improve the forming precision and the surface quality of the parts, but also provide the possibility for the processing and forming of thin-walled and complex-shaped parts $[4,5]$.

In recent years, the fluid pressure forming process has become one of the important forming technology methods for complex deep drawing parts [6]. Professor Yuan SJ et al. [7, 8] proposed a new fluid hydroforming process for the difficulty of forming thin-walled curved parts, and established a theoretical model of fluid pressure forming. The loading path was changed by using fluid pressure for bulging in the early stage of deep drawing, and formed a 3m-level integral thin-walled curved surface component successfully. Liu W et al. [9] used the method of combining bulge pre-forming and hydroforming technology to study the stress distribution and deformation mechanism of the parts. The results showed that the pre-bulging process can change the stress state of the material and improve the uniformity of deformation. Li WD et al. [10] studied the influence of changing the pre-forming parameters and process path on the deformation sequence of aluminum alloy tapered deep-drawn parts, and analyzed the results 
of numerical simulations to produce the tapered parts with uniform thickness distribution and high dimensional accuracy. Liu W et al. [11] studied the influence of pre-bulging pressure and cavity pressure on the forming of parts in combination with numerical simulation and experiment. The study found that reducing the tangential compressive stress of the deformed material in unsupported wall region and increasing the contact area between the material and the punch can eliminate wrinkle defects effectively. Lang LH et al. [12] studied the influence of the pre-forming effect on the forming of the box-shaped parts during the hydroforming process. The research results showed that too high and too low pre-bulging height are not conducive to forming, and too large pre-bulging pressure will cause obvious cracks and wrinkles in the punch part. Furthermore, the influencing factors of the pre-forming effect in the hydroforming process of tapered parts are explored, they found that a reasonable initial inverse bulge height and pressure can make the wall thickness of the part uniform and effectively reduce the wall thickness reduction rate [13].Chen BG et al. [14] quantitatively described the influence of the amount of pre-bulging on the hydraulic deep drawing of cylindrical parts through a combination of experiment and numerical simulation, and a certain amount of pre-bulging can increase the deformation and the hardness of the bottom of the cylindrical part.

MR fluid is a new type of intelligent material, its rheological properties will change under the action of an external magnetic field. The magnetic particles are regularly arranged along the direction of the magnetic field to form a chain-like structure. With the increase of squeezing force, the chain of magnetic particles will break and reorganize continuously to form more stable stone clusters. Professor Merklein et al. [15] studied the application of MR fluid in the field of sheet metal forming for the first time, and MR fluid was used in the hydraulic deep drawing process as a sealing ring to increase the forming limit of the sheet. Professor Wang ZJ $[16,17]$ of Harbin Institute of Technology has conducted a series of researches on the forming process of MR fluid soft mold. MR fluid is used as a punch for bulging, which is different from other soft mold medium, MR fluid has a magnetorheological effect and can change the stress state of the sheet metal in the deformation area by controlling the intensity of the external magnetic field. Wang PY et al. [18] studied the mechanism of the influence of variable magnetic field conditions on the bulge forming performance of metal sheets, and the research indicated that under the external magnetic field conditions, the forming properties of the sheet metal were significantly improved. Wen Tong et al. [19] used MR fluid to form high-precision tubular parts. With the increase of the magnetic field strength, the uniformity of the wall thickness distribution of the pipe parts increased, and the overall forming quality was significantly improved. Xu Peng [20] and others further studied the application of MR fluid in the sheet deep drawing process. The study showed that under the conditions of MR fluid assisted deep drawing, the forming performance of the part was significantly improved and the transitional thinning of the wall thickness at the corners of the punch is suppressed, which broadens the way for the application of magnetic medium in the field of sheet metal forming.

Therefore, this article uses MR fluid as a flexible forming medium in the deep drawing process of sheet metal. Under the action of an external magnetic field, the influence of the pre-forming process on the forming performance of the cylindrical part is studied by changing the height of the inverse bulge preforming, and inverse bulge deep drawing composite experiments were conducted under different pre- 
forming conditions. The experimental results are verified by finite element numerical simulation, and the mechanism of the influence of MR fluid inverse bulge pre-forming effect on the forming quality of cylindrical parts under different forming conditions is further analyzed.

\section{Research Program}

\subsection{Experimental Materials}

The blank for the research is an Al5052 circular sheet with a thickness of $1 \mathrm{~mm}$, and the uniaxial tensile test experiment was finished by the electronic universal material testing machine. The tensile samples were taken at $0^{\circ}, 45^{\circ}$, and $90^{\circ}$ along the rolling direction of the plate, and the true stress-strain curves of the plates with different orientations were measured as shown in Fig. 1. Assuming that the true stressstrain curve of 5052 aluminum alloy conforms to the relationship, where is the strength factor and is the strain hardening exponent.Take the logarithm of both sides of the formula to get, and the strain hardening exponent of the material is obtained by fitting the true stress-strain relationship curve of 5052 aluminum alloy. The mechanical properties of the Al5052 sheet are shown in Table 1. It can be seen that the stress-strain curves of the Al5052 sheet along the directions of $0^{\circ}, 45^{\circ}$, and $90^{\circ}$ are different. In order to reduce the error, we take the average value of the mechanical properties of the materials in each direction.

Table 1

Mechanical properties of 5052 aluminum alloy sheet

\begin{tabular}{|llllll|}
\hline Direction & Yield Strength /MPa & $\begin{array}{l}\text { Tensile strength } \\
\text { /MPa }\end{array}$ & Elongatio /\% & $n$ & $\mathrm{~K}$ \\
\hline $0^{\circ}$ & 99 & 197.1 & $23.2 \%$ & 0.37 & 400.58 \\
\hline $45^{\circ}$ & 91.8 & 187.3 & $22.4 \%$ & 0.32 & 370.71 \\
\hline $90^{\circ}$ & 94 & 196.6 & $22 \%$ & 0.33 & 353.71 \\
\hline Average value & 95 & 193.7 & $22.53 \%$ & 0.34 & 375 \\
\hline 2.2 Research program & & & & \\
\hline
\end{tabular}

The experiment was conducted on a $500 \mathrm{KN}$ hydraulic workbench with a loading speed of $0.5 \mathrm{~mm} / \mathrm{s}$. A round Al5052 sheet was used for the experiment, and the thickness is $1 \mathrm{~mm}$, the diameters are $85 \mathrm{~mm}$ and $90 \mathrm{~mm}$ respectively. Define $\mathrm{K}=\mathrm{D} / \mathrm{d}$ as the drawing ratio, the drawing ratios of the formed parts are $\mathrm{K}_{1}=$ 2.125 and $\mathrm{K}_{2}=2.25$ respectively. The experiment adopts a fixed-gap blanking method, in which blanking gap is $0.2 t$, and that between convex and concave die is $1.2 t$. The MR fluid flexible medium is filled with a closed space composed of a concave mold cavity, a back pressure unit and a sheet material. The schematic diagram of the experimental principle of inverse bulge deep drawing composite experiments with MR fluid is shown in Fig. 2. The experimental device is mainly composed of a punch, a blank holder, a concave die, a back pressure unit and a magnetic field control unit. The external magnetic field is 
generated by a magnetic field conrrol unit formed by a number of turns of a coil wound on the outside of the cavity mold. The power supply device, sliding rheostat and ammeter are connected in series, and the strength of the magnetic field is controlled by the magnitude of the current.

This study will be divided into two groups, the diameters of the blanks selected for the experiment are $85 \mathrm{~mm}$ and $90 \mathrm{~mm}$ respectively. In each group of experiments, including conventional deep drawing forming, MR fluid soft mold forming and MR fluid inverse bulge deep drawing composite forming, and the height of inverse bulge pre-forming are $5 \mathrm{~mm}, 7 \mathrm{~mm}, 9 \mathrm{~mm}$, and $11 \mathrm{~mm}$ respectively.

Figure 2 Principle of inverse bulge deep drawing composite forming (a) Schematic diagram of experimental device (b) Experimental equipment (c) Magnetic field generating device (d) Physical state diagram of MR fluid when $B=0 T$ (e) Physical state diagram of MR fluid when $B=0.1 T$

\subsection{Process analysis}

Figure 3 shows the process of using MR fluid soft mold medium for inverse bulge deep drawing composite forming. This forming process is mainly completed in two stages, the inverse bulging preforming is carried out firstly, and then conducted the deep drawing final forming. The whole forming process is conducted in a uniform magnetic field with a magnetic field strength of $0.1 T$. The MR fluid will have a magnetorheological effect under the action of an external magnetic field, and the magnetic particles are arranged in a chain structure along the direction of the magnetic field. In the initial stage of forming, the back pressure unit moves upward to load, and the MR fluid is regarded as a pressure transfer medium to transfer pressure under the action of back pressure to deform the sheet. When the height of the inverse bulge pre-forming reaches a predetermined height, the back pressure unit moves in the opposite direction to cooperate with the punch to load downwards. At this time, under the action of the bidirectional pressure of the punch and the magnetic medium, an aluminum alloy flat-bottomed cylindrical part is finally formed after four stages of pressing down, flattening, early drawing stage and final drawing stage. During the forming process, the MR fluid flexible medium always acts on the surface of the sheet under the pressure of the back pressure unit, forcing the sheet to close to the punch for deformation, and the soft drawbead is formed at the gap between punch and blank holder in the process of sheet pressing.

\subsection{Finite element simulation}

As shown in Fig. 4, the finite element model of inverse bulge and deep drawing composite forming is established by using Dedorm-2D commercial software. Since the cylindrical part has axisymmetric geometric characteristics, and 1/2 axisymmetric part is selected for model establishment. The punch, blank holder, die and piston are set as rigid model, and the sheet and MR fluid are rigid plastic model, and they are meshed. The sheet is divided into 750 elelments and the MR fluid is divided into 5000 elements, and the boundary conditions are applied to the MR fluid and the sheet along the $X$ axis to limit the 
deformation. As shown in Fig. 1, the flow stress of the Al5052 sheet has been obtained in the tensile experiment. and the true stress-strain curve obtained from the experiment is applied to the establishment of the material model. The MR fluid with a mass solid content of $56 \%$ was selected in the experiment, and the rheological stress parameters were set according to the constitutive model of the MR fluid that has been studied in the literature [21]. The punch radius and the die radius is $5 \mathrm{~mm}$ and $6 \mathrm{~mm}$ respectivily, and the radius of the blank holder is $8 \mathrm{~mm}$. In order to improve the accuracy of the finite element simulation results about the influence of the MR fluid on the sheet deformation, the meshes of the MR fluid plastic model was locally refined. Table 2 shows the friction conditions between the sheet and the MR fluid and the rigid mold. The loading speed of the back pressure unit along the $Y$-axis during the bulging process is $1 \mathrm{~mm} / \mathrm{s}$, and the punch along the $Y$-axis downward loading speed is $0.5 \mathrm{~mm} / \mathrm{s}$ during the deep drawing process.

Table 2

Interface friction parameters of finite element simulation

\begin{tabular}{|ll|}
\hline Contact surface & Coefficient of friction \\
\hline Blank/Punch & $\mu=0.5$ \\
\hline $\begin{array}{l}\text { Blank/Blank holder } \\
\text { Blank/Die }\end{array}$ & $\mu=0.1$ \\
Blank/MRF & $\mathrm{m}=0.1$ \\
Die/MRF & \\
Piston/MRF & \\
\hline Piston/Die & $\mathrm{m}=0.12$ \\
\hline
\end{tabular}

\section{Results And Discussion}

\subsection{Wall thickness distribution}

Under different forming conditions, the cylindrical parts with the drawing ratios are $\mathrm{K}_{1}=2.125$ and $\mathrm{K}_{2}=$ 2.25 respectively are shown in Fig. 5. After the experiment is completed, as shown in Fig. 6. measuring the wall thickness distribution of the two kinds of drawing ratios of cylindrical parts, and calculating the variation of the wall thickness. According to the distribution position of the measuring points, the cylindrical part can be divided into three deformation areas along the central axial section, are at the

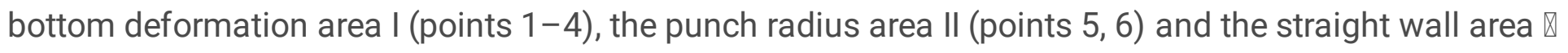
(Points 7-13, 14) respectively.

Figure $6 a) \square b$ ) show the wall thickness distribution of cylindrical parts with different drawing ratios under different forming conditions. We can find that the wall thickness at the bottom of the cylinder and the measuring points near the punch corner area are thinned after deep drawing, where the wall thickness of 
the punch corner area thining seriously and the straight wall area (such as measuring points 10-14) thickening. As shown in Fig. 6, compared with the wall thickness distribution of conventional deep-drawn parts, the uniformity of the wall thickness distribution of the parts have improved after using the MR fluid flexible medium for forming, and the wall thickness thining trend near the bottom area and thickening trend near the punch corner area of the cylinder parts are both decreaseed. In the inverse bulge and deep drawing composite forming process, the MR fluid acts as a force transmission medium to change the stress state of the sheet metal and suppress the occurrence of wrinkling defects effectively. With the increase of the inverse bulge height, the thinning of wall thickness decreases at the bottom and the punch corner area of the cylindrical parts. The thickening trend of the side wall of the cylindrical part is slowed down, and the distribution of the wall thickness is more uniform. However, when the pre-forming height is too high, the thickness of the bottom of the cylinder part becomes thinner because of the inverse bulge forming, where the material is enriched. The cylindrical wall was thicken and the wall thickness of the straight wall near the mouth of the cylindrical part is the most serious.

Calculate the chang rate of wall thickness at each measuring point of the cylindrical part with different drawing ratios, as shown in Fig. 6c) $\square$ d). It can be found that with the conventional drawing process conditions, the wall thickness of the punch fillet area of the formed part is thinned seriously and the maximum thinning rate are $10.1 \%$ and $11.3 \%$ with the drawing ratio of $\mathrm{K}_{1}=2.125$ and $\mathrm{K}_{2}=2.25$ respectively, the wall thickness of the straight wall area is thicken robviously, and the maximum thickening rate are $24.1 \%$ and $21.2 \%$ respectively. Compared with conventional deep drawing conditions, the maximum wall thickness thining rate at the punch corner area of the cylindrical part formed with the MR fluid soft medium has been reduced by $8.9 \%$ and $22.12 \%$ respectively, and the maximum wall thickness thickening rate of the cylinderical wall area is reduced by $11.6 \%$ and $12.7 \%$ respectively. The maximum wall thickness thining rate near the bottom center area (point 1 ) decreased by $36.4 \%$ and $27.7 \%$ respectively. The inverse bulge pre-forming process has a significant influence on the wall thickness distribution of the formed part. With the increase of the inverse bulge height, the wall thickness increase rate and thinning rate at different measurement points are reduced, and the overall forming performance is improved. The inverse bulge pre-forming process has a significant influence on the wall thickness distribution of the parts. With the increase of the inverse bulge height, the thickening and thinning rate of the wall thickness at different measurement points are reduced, and the overall forming performance of the cylinderical part has been improved. For cylindrical parts with drawing ratio $\mathrm{K}_{1}=$ 2.125 , when the inverse bulge height is $9 \mathrm{~mm}$, the maximum thining rate of the bottom wall thickness of the cylindrical part is $3.7 \%$, and the maximum thining rate of the wall thickness at the punch corner area is $5.2 \%$, and the maximum thickening rate at the straight wall area is $6.1 \%$. For cylindrical parts with drawing ratio $\mathrm{K}_{2}=2.25$, when the inverse bulge height is $5 \mathrm{~mm}$, the maximum wall thickness thining rate at the bottom area and the punch corner area of the cylinderical part is $2.9 \%$ and $9.1 \%$ respectively, and the maximum thickening rate at the straight wall area is $6 \%$. Reasonable height of inverse bulge can suppress the phenomenon of wall thickness thining and thickening of the cylindrical part effectively. When the inverse bulge height is $11 \mathrm{~mm}$, the wall thickness of the bottom area of the cylindrical part with different drawing ratios are thinned extremely, extreme point appears near the punch corner area (point 7), 
where the thinning rates are $13.1 \%$ and $14.4 \%$ respectively. When the height of the inverse bulge preforming is lower, which is beneficial to the forming of the drawn part and the better the performance of the formed part.

\subsection{Wall thickness change rate}

As shown in Fig. 7, Comparing and analyzing the maximum change rate of wall thickness and the variance of the wall thickness of cylindrical parts with different drawing ratios. Figure 7a) shows the maximum thinning rate and thickening rate of the wall thickness of cylindrical parts under different forming conditions. The comparison of the wall thickness change rates of cylindrical parts with drawing ratios of $\mathrm{K}_{1}=2.125$ and $\mathrm{K}_{2}=2.25$ respectively, When the MR fluid assisted deep drawing, the maximum wall thickness thining rates are $9.2 \%$ and $10.6 \%$ respectively. Compared with the conventional drawing conditions, which are reduced by $8.9 \%$ and $6.19 \%$ respectively. The maximum wall thickness thining values appear at measurement points 5 and 7 respectively. The maximum wall thicknesst thickening rates are $21.3 \%$ and $18.5 \%$ respectively, which are reduced by $11.6 \%$ and $12.7 \%$, and the maximum wall thickness thickening values appear at the measurement points 13 and 14 respectively.It can be found that a reasonable amount of inverse bulge pre-forming will increase the maximum wall thickness thining rate of the cylindrical parts, and with the increase of the inverse bulge height, the maximum wall thickness thining rate will decrease. However, when the inverse bulge height is $11 \mathrm{~mm}$, the maximum wall thickness thining rate increases obvisouly. When the drawing ratio $\mathrm{K}_{1}=2.125$, the maximum wall thickness thickening rate decreases with the increase of the inverse bulge height, and it will decreases to $6.1 \%$ as the inverse bulge height is $9 \mathrm{~mm}$. The maximum wall thickness thickening rate is the smallest, which is reduced to $6 \%$, when the inverse bulge height is $5 \mathrm{~mm}$ for the drawing ratio of the parts is $\mathrm{K}_{2}=$ 2.25 , and as the inverse bulge height increases, the maximum wall thickness thickening rate increases.

Figure 7b) shows the mean value and variance of the wall thickness of the cylindrical parts with different drawing ratios under different forming conditions. The MR fluid medium is used for deep drawing. After the MR fluid is used for assisting deep drawing, the wall thickness variance of the cylindrical parts is compared with the conventional deep drawing, which are reduced by $40.8 \%$ and $37.2 \%$ respectively, and the uniformity of the wall thickness is improved. When the drawing ratio is 2.125 , the mean value and variance value are continuously reduced with the increase of the inverse bulge height. When the inverse bulge height is $9 \mathrm{~mm}$, the variance value decreases by $86.31 \%$, reduced to 0.0023 . When the drawing ratio is 2.25 , the wall thickness distribution is more uniform under the smaller inverse bulge pre-forming height, and the variance value is about 0.0025 as the inverse bulge height is $5 \mathrm{~mm}$, which is reduced by $82.8 \%$. With the height of inverse bulge pre-forming increaseing, the variance value keeps increasing. When the inverse bulge height increases by $11 \mathrm{~mm}$, the wall thickness variance of the two kinds of drawing ratios of the cylindrical parts increases sharply and the uniformity of the wall thickness distribution is lower, that is the excessive inverse bulge height is not favorable to the deep drawing. When the drawing ratio is 2.125, the best pre-forming height is about $9 \mathrm{~mm}$. The best pre-forming height is constantly decreasing as the drawing ratio increase, this is because the large-diameter blank has a larger contact area with the flange area, which will increase the frictional force. And the inverse bulge deformation will reduce the thickness 
of the blank, resulting in the material is more difficult to flow. For cylindrical parts with a drawing ratio of 2.25 , the best inverse bulge height is about $5 \mathrm{~mm}$.

\subsection{Equivalent stress distribution after inverse bulge pre-forming}

Under different forming processes, the equivalent stress distribution of the cylindrical parts with two drawing ratios after inverse bulge pre-forming is shown in Fig. 8 . It is defined that the side of the sheet material close to the blank holder is the outside, and the side close to the die is the inside. During the inverse bulge pre-forming process, the outside of the blank at the fillet of the blank holder is stretched, and the inner side of the bulging center area of the sheet is compressed, and the outer side is stretched. As shown in Fig. 8a) and Fig. 8b), when the inverse bulge height is $7 \mathrm{~mm}$, the material in the bulging deformation area becames yield almost at the same time. Under the combined action of the MR fluid and the blank holder, plastic deformation has occurred around the fillet area of the blank holder. The maximum equivalent stress value exists on the inside of the sheet, and the minimum value appears on the inside of the transition area between the fillet area and the free bulging forming area. The equivalent stress has a stress gradient along the thickness direction in the deformation area. As the height of the inverse bulge increases, the plastic deformation area of the rounded corner area expands continuously and the stress gradient along the thickness direction increases, the deformation begins to move to the center of the bulging forming area. When the inverse bulge height is $11 \mathrm{~mm}$, the maximum equivalent stress exists on the outer surface of the highest point of the bulging area. The circumferential stress and radial stress of the bulging center area are basically equal, which is obvious two-way tensile stress state, and the wall thickness is severely thinned. As shown in Fig. 8c), the maximum equivalent stress of cylindrical parts with different drawing ratios in the pre-forming stage increases with the height of the inverse bulge increase, and the difference value of the maximum equivalent stress decreases firstly and then increases. When the height of the inverse bulge pre-forming is the same, the cylindrical parts with a drawing ratio of 2.125 enters the deformation state earlier than the drawing ratio of 2.25 , the plasic deformation area is larger and the inverse bulge effect is more obvious.

\subsection{Stress analysis of deep drawing}

The cylindrical part during deep drawing forming is shown in Fig. 9. We divide the cylindrical part into five parts: flange area, die corner area, straight wall area, bottom corner area and bottom area, and the side close to the punch is the inner side of the cylinderical wall, and the side close to the die is the outer side of the cylinderical wall. Under the action of an external magnetic field, the aluminum alloy after inverse bulge pre-forming is deep-drawn forming with soft die using MR fluid medium, and analyzed the stress and strain state of cylindrical parts under different forming processes.

Since the inverse bulge pre-forming process has a significant effect on the drawing performance and stress state of the cylindrical part, we take measuring points along the outer area of the wall in order to further study the influence of the inverse bulge pre-forming effect on the deep drawing process. In the early stage of the deep drawing forming, the inverse bulge area appears obvious and the sheet at the corner of the die forms a soft drawbead. As the drawing displacement increases, the inverse bulge pre- 
forming area is reduced gradually. When the drawing displacement is about $12 \mathrm{~mm}$, the inverse bulge deformation area basically disappears. And the sheet is drawn into the die with the increase of the drawing displacement.

Figure 10a) shows the maximum radial stress of the cylindrical parts under different forming conditions at the early stage of deep drawing (the displacement of the punch is $12 \mathrm{~mm}$ ). During the deep drawing process, the deformation of the plate at the flange fillet is the most severe, and stress concentration occurs in the inner area of the sheet at the transition between the die corner and the flange area, where the maximum radial tensile stress occurs. Meanwhile, the wrinkling defect here is the most difficult to overcome, and the tendency of wrinkling at the fillet can be suppressed by improving the fluidity of the material in the flange area. In the case of using the MR fluid as the soft mold medium for forming, the maximum tensile stress values of the cylindrical parts with drawing ratios of 2.125 and 2.25 are smaller than that under conventional drawing conditions. At the early stage of deep drawing forming, the radial tensile stress value of the sheet in the flange decreases and the absolute value of the radial compressive stress increases under the action of back pressure, which is beneficial for the material to flow into the cavity of the die. Therefore, the tendency of wrinkling defects and lug phenomenon is suppressed effectively, and the surface quality of the side wall is better. When the inverse bulge height is $5 \mathrm{~mm}$, the amount of material deformation in the fillet area is small and the effect of the soft drawbead is weaker, the maximum radial tensile stress value decreases and the compressive stress value increases. The soft drawbead has a significant effect after pre-forming with the increase of the deformation height. and the friction drag and resistance to deformation increaseing accordingly after the material at the die corner area has strain strengthening after the bending and anti-bending deformation processes.

When the drawing displacement is the same, the pre-forming effect has a similar influence on the material deformation of the flange area of the cylindrical part with different drawing ratios. Therefore, the stress state of the sheet material in the flange area of the cylindrical part with a drawing ratio of $\mathrm{K}_{1}=$ 2.125 is analyzed. In the entire inverse bulge and deep drawing forming process, the deformation of the materials in each area of the cylindrical part affects each other. The fluidity of the sheet matel in the flange area is very critical to the quality of the final formed part. The flow resistance of the material can be adjusted to control its uniformity of deformation.

In the early stage of deep drawing process, the circumferential stress distribution of the sheet metal in the flange area under different forming conditions is shown in Fig. 10 (b). It can be seen that the material in the flange area is subjected to circumferential compressive stress, and the sheet material is easy to lose stability and wrinkle. In the initial stage of the conventional deep drawing forming, the circumferential compressive stress in the flange area is relatively large. With the assistance of the magnetic mediam, the back pressure can be transmitted through the magnetic particles, so that the sheet metal is close to the surface of the punch, and the friction resistance is increased. The circumferential compressive stress value near the corner of the die increases. At the same time, the MR fluid has a lubricating effect, which reduces the friction between the sheet material and the surface of the die, increases the fluidity of the sheet material, and effectively reduces the circumferential compressive stress in the flange area. 
However, the circumferential stress distribution of the sheet metal in the flange area is extremely uneven, and wrinkling defects are obvious during these two forming processes. After inverse pre-forming, a soft drawing bead is formed near the corner of the die, which increases the flow resistance of the sheet in the flange area and the amount of circumferential deformation, and reduces the value of compressive stress. And with the increase of the inverse bulge height, the surface area of the sheet material used for the bottom forming of the cylindrical member gradually expands, and the suspended area increases accordingly, as shown in Fig. 11. The area of the suspended area under different forming processes is $A$, $B, C$ and $D$, that is, $A<B<C<D$, the area of the soft draw bead is enlarged during the deep drawing process, and the plastic deformation ability of the sheet metal is improved. When the inverse bulge height is $5 \mathrm{~mm}$, the radial tensile stress near the corner of the die increases, and the circumferential compressive stress decreases. As the height of inverse height increases, the value of the circumferential compressive stress at the corner of the die increases, the distribution of compressive stress in the flange area is gradually uniform. When the inverse bulge height is about $9 \mathrm{~mm}$, the distribution of the compressive stress in the flange area is the most uniform, and the formation of the soft draw bead increases the area of the sheet metal against the punch and improves the forming performance of the cylindrical part. When the inverse bulge height is $11 \mathrm{~mm}$, the circumferential compressive stress value is too larget to cause wrinkling.

In the early stage of deep drawing, there is bending of the sheet at the corners of the die-reverse bending is caused by the effect of pre-forming deformation, and the inner and outer sides of the sheet are plastically deformed, and the stress has a stress gradient along the thickness. Analyzing the cylindrical parts with a drawing ratio of $\mathrm{K} 1=2.125$, as shown in Fig. 12 , study the radial stress and equivalent stress distribution along the thickness direction at the fillet of the deep part die under different inverse bulge forming conditions. The inner side of the cylinder wall is the radial tensile stress and the outer side is the radial compressive stress, and there is a stress gradient along the thickness direction, the greater the radial tensile stress is, the more likely the fracture occurs. In the conventional deep drawing process, the maximum radial tensile stress at the fillet is $188 \mathrm{Mpa}$, the maximum compressive stress is $48.2 \mathrm{Mpa}$, and the stress gradient is $236.2 \mathrm{Mpa}$, where the equivalent stress gradient is large, and the plastic deformation at the fillet is obvious. In the process of MR fluid soft die forming, the deformation at the corner of the die is small under the back pressure of the magnetic medium. At this time, the radial tensile stress and compressive stress are smaller, the radial stress gradient and equivalent strain gradient decrease. After the inverse bulge and deep drawing composite forming, due to the effect of soft drawbead in the early stage of deep drawing, the outer tensile stress and inner compressive stress decrease and the radial stress gradient decreases when the reverse bulging height is small. As the amount of pre-deformation increases, the tensile stress value at the outside of the sheet increases, and the compressive stress value at the inside decreases. When the inverse bulge height is about $9 \mathrm{~mm}$, the outside tensile stress is $187 \mathrm{Mpa}$, and the inside compressive stress is $1.6 \mathrm{Mpa}$, the stress gradient is $188.6 \mathrm{Mpa}$. The stress neutral layer has moved, the radial compressive stress on the inner side turns into tensile stress. The equivalent stress gradient is the smallest and the material is easy to flow under tensile stress. When the inverse bulge height is $11 \mathrm{~mm}$, the tensile stress decreases along the thickness direction continuously, and the 
inner side of the sheet changes from compressive stress to tensile stress, and the stress gradient decreases. However, the bottom of the cylinder becomes in a cone after deep drawing due to the excessive pre-deformation and insufficient back pressure, and the forming effect is not good.

\section{4】 Conclusion}

1. This paper proposed a composite forming process of inverse bulge pre-forming and deep drawing of cylindrical parts. The smart material of MR fluid is used to transfer pressure under the external magnetic field. In the pre-forming stage, the MR fluid is used as the punch to bulge, and in the final forming stage, the MR fluid is used as the back pressure unit to form the deep drawing parts. When the inverse bulge height is about $9 \mathrm{~mm}$ and $5 \mathrm{~mm}$, the forming quality of the cylindrical parts with the two drawing ratios is the best, while the inverse bulge height is too high or too low, the wrinkle defect cannot be suppressed effectively.

2. For the two kinds of drawing ratios of cylindrical parts, we completed respectively the experiments under different forming conditions. The results of the research show that the variance of the wall thickness of the formed part is reduced by $40.8 \%$ and $37.2 \%$, respectively as using MR fluid soft mold media for deep drawing. Reasonable pre-bulging height is conducive to improving the uniformity of wall thickness distribution. When the inverse bulge height is $9 \mathrm{~mm}$ and $5 \mathrm{~mm}$, the wall thickness variance of the two drawing ratios of cylindrical parts is the smallest, which is 0.0023 and 0.0025 respectively.

3. A finite element analysis model was established to analyze the equivalent stress distribution in the pre-forming stage under different forming conditions. the sheet near the fillet area of the blank holder occurs plastic deformation firstly, and the maximum equivalent stress increases with the increase of the inverse bulge height, and the plastic deformation area increases significantly in the meanwhile. When the inverse bulge height is $11 \mathrm{~mm}$, the maximum equivalent stress is on the outer surface of the highest of the bulging area. When the pre-forming height is the same, the smaller of the drawing ratio is, the pre-forming effect is more obvious.

4. The pre-forming effect will affect the distribution of sheet stress significantly. Take a part with a drawing ratio of $\mathrm{K}_{1}=2.125$ as an example, the maximum radial stress in the flange area increases with the increase of the pre-forming height under the action of back pressure and soft drawbeads at the initial stage of deep drawing, while the compressive stress is reduced. The radial stress gradient and the equivalent stress gradient at the corners of the die are reduced. With the increase of the preforming height, the distribution of the circumferential is uniform. When the inverse bulge height is about $9 \mathrm{~mm}$, the stress gradient is small, and the larger radial tensile stress and circumferential compressive stress can suppress the wrinkle.

\section{Declarations}

\section{Ethical Approval}


Not applicable

\section{Consent to Participate}

Not applicable

\section{Consent to Publish}

Not applicable

\section{Authors Coutributions}

The development of flexible media forming technology can improve the forming precision and the surface quality of the parts and provide the possibility for the processing and forming of thin-walled and complex-shaped parts.

\section{Funding information}

This paper was financially Supported by the Key Laboratory of Micro-systems and Micro-structures Manufacturing of Ministry of Education, Harbin Institute of Technology and the Natural Science Foundation of Heilongjiang Province (LH2019E056).

\section{Competing Interests}

Not applicable

\section{Availability of data and materials}

The data obtained in the framework of this study

are available to the journal upon request.

\section{References}

1. Abd El-AtyA, Xu Y, Guo XZ, et al. (2018) Strengthening mechanisms, deformation behavior, and anisotropic mechanical properties of Al-Li alloys: A review. Journal of Advanced Research, 10: 49-67

2. Choi H, Koç M, Ni J (2007) A study on the analytical modeling for warm hydro-mechanical deep drawing of lightweight materials. International Journal of Machine Tools and Manufacture, 47:17521766

3. Yuan S, He Z, Liu G (2012) New developments of hydroforming in China. Materials Transactions, 53(5): 787-795

4. Ray P, Donald BJM (2005) Experimental study and finite element analysis of simple X- and T-branch tube hydroforming processes. International Journal Mechanical Science, 47(10):1498-1518 
5. Mori K, Patwari AU, Maki S (2004) Improvement of formability by oscillation of internal pressure in pulsating hydroforming of tube. CIRP Annals-Manufacturing, 53(1):215-218

6. Yuan SJ\&Fan XB (2019) Developments and perspectives on the precision forming processes for ultra-large size integrated components. International Journal of Extreme Manufacturing, 1(2):022002

7. Chen YZ囚Liu WखZhang ZC『et al. (2017) Analysis of wrinkling during sheet hydroforming of curved surface shell considering reverse bulging effect. International Journal of Mechanical Sciences, 20:7080

8. Chen YZ, Liu W, Xu YC, et al. (2015) Analysis and experiment on wrinkling suppression for hydroforming of curved surface shell. International Journal of Mechanical Sciences, 104:112-125

9. Liu W, Chen YZ, Xu YC, et al.(2018) Enhancement on plastic deformation of curved surface shell by sheet hydroforming with optimized pre-bulging process. International Journal of Advanced Manufacturing Technology, 97(9-12):4145-4156

10. Li WD, Meng B, Wang C, Wan M, Xu L (2017) Effect of prebulging and pressure path on deformation behavior in multi-pass hydrodynamic deep drawing process. International Journal of Mechanical Sciences, 121:171-180

11. Liu W, Xu Y, Yuan S (2014) Effect of pre-bulging on wrinkling of curved surface part by hydromechanical deep drawing. Procedia Engineering, 81:914-920

12. Lang LH, Wang YM, Xie YS. et.al. (2012) Pre-bulging effect during sheet hydroforming process of aluminum alloy box with unequal height and flat bottom. Transactions of Nonferrous Metal Society of China, 22:302-308

13. Lang LH, Zhang QD. (2017) Influence Law of Initial Reverse Bulging on Sheet Hydroforming Process. Key Engineering Materials, 746: 99-107

14. Chen BG, Xu YC. (2011) Influence of pre-bulging on deformation and hardness of dual-phase steel cylindrical cup by hydrodynamic deep drawing process. Materials Science \& Technology, 9(1):17-19

15. Rosel S, Merklein M, (2014) Improving formability due to an enhancement of sealing limits caused by using a smart fluid as active fluid media for hydroforming. Production Engineering, 8(1-2): 7-15

16. Wang ZJ, Xiang N, Wang PY, Li ZX. (2017) Property-adjustable forming media induced extension of sheet metal formability under variable magnetic field. Journal of Materials Processing Technology, 243: $420-432$

17. Wang PY, Wang ZJ, Xiang N. et al. (2020). Machanism of magnetic field condition on deformation behavior of sheet metal using a property-adjustable flexible-die. International Journal of Advanced Manufacturing Technology, 109(3-4): 629-644

18. Wang PY, Wang ZJ, Xiang N. (2020) Investigation on changing loading path in sheet metal forming by applying a property-adjustable flexible-die. Journal of Manufacturing Processes, 53: 364-375

19. Wen T, Zhang ZS, Zhang M et al. (2018). Precision bending of thin-walled tubes based on inserted magnetic rheological particles as infill. Journal of Huazhong University of Science and Technology, 46(7):63-66 
20. Li F, Xu P, Sui XC, Zhou FJ. (2015) Investigation of a New Method for Sheet Deep Drawing Based on the Pressure of Magnetic Medium[J]. Materials Transactions, 56(6):781- 784

\section{Figures}

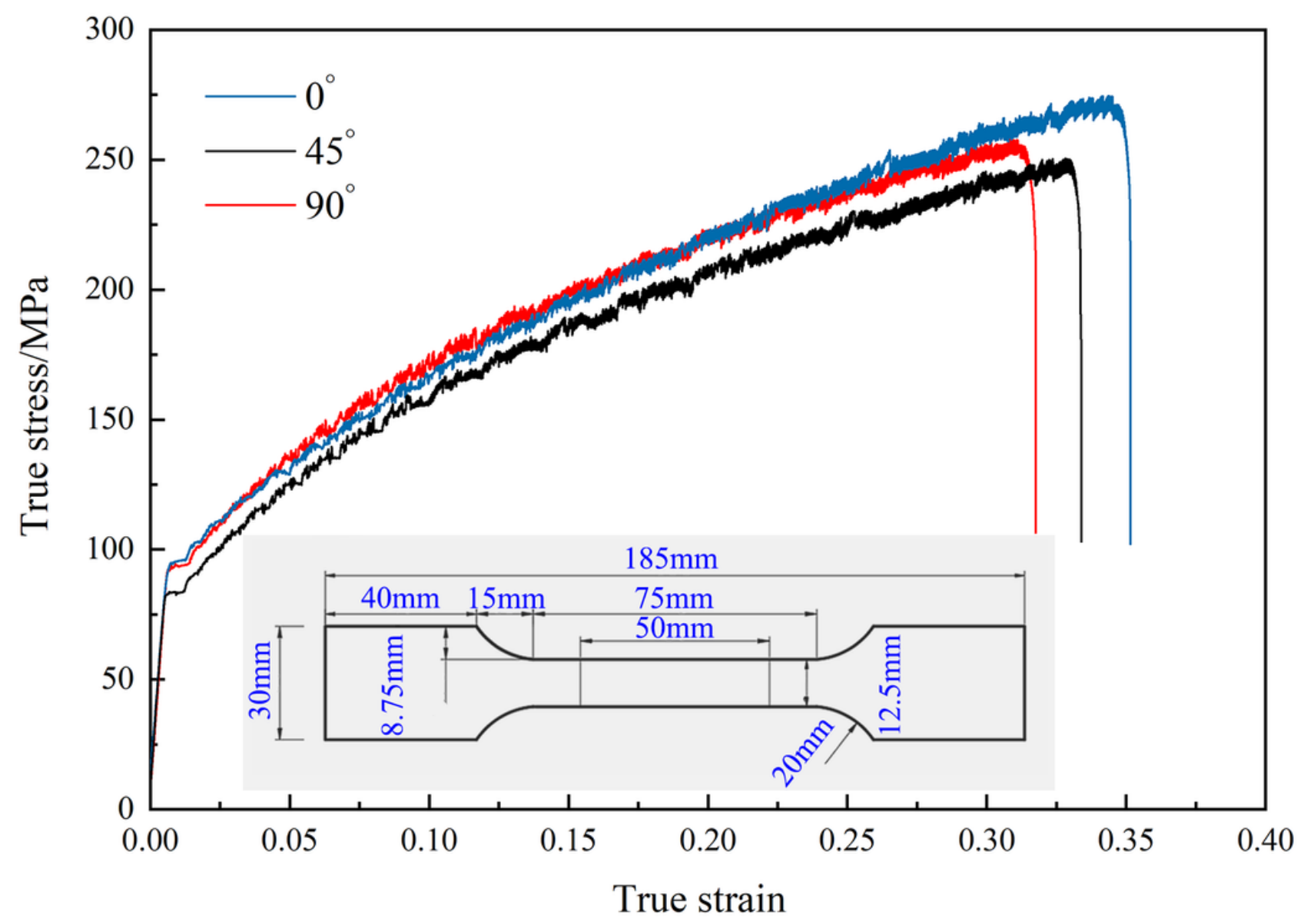

Figure 1

The true stress-strain curves of materials with different rolling directions 


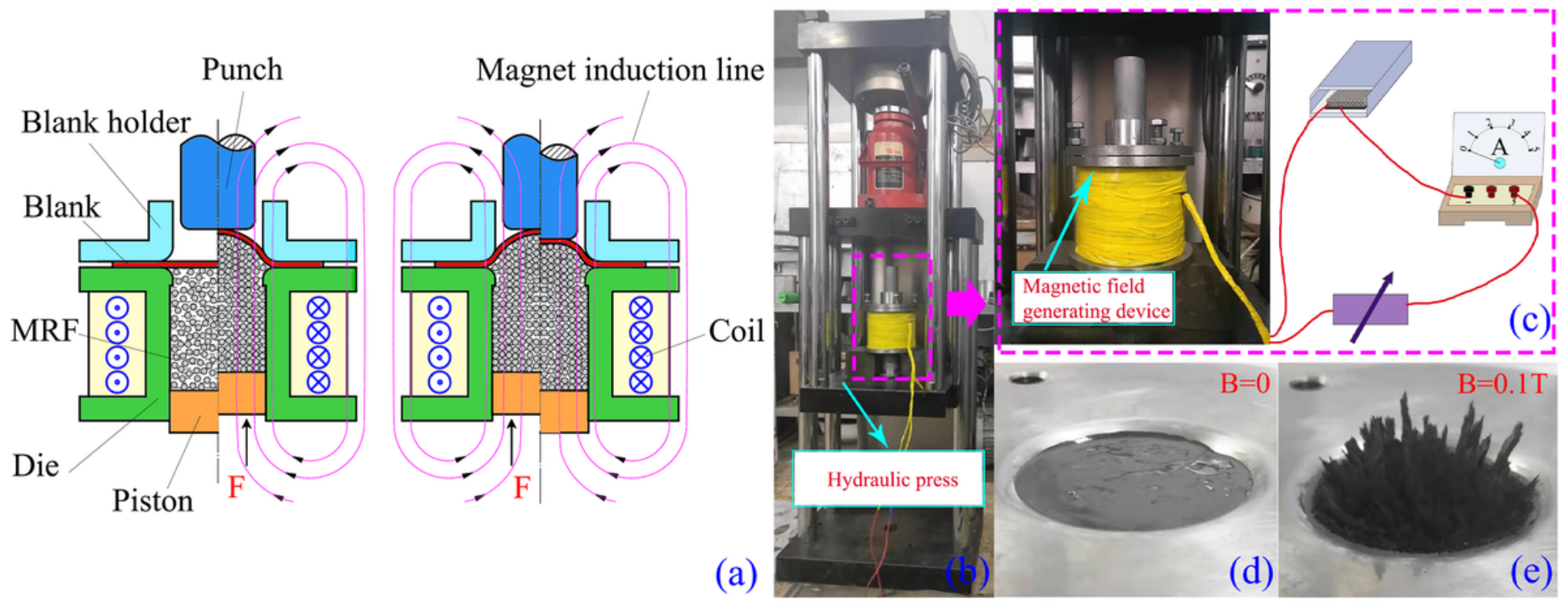

Figure 2

Principle of inverse bulge deep drawing composite forming (a) Schematic diagram of experimental device (b) Experimental equipment (c) Magnetic field generating device (d) Physical state diagram of MR fluid when $B=0 T(e)$ Physical state diagram of MR fluid when $B=0.1 T$ 

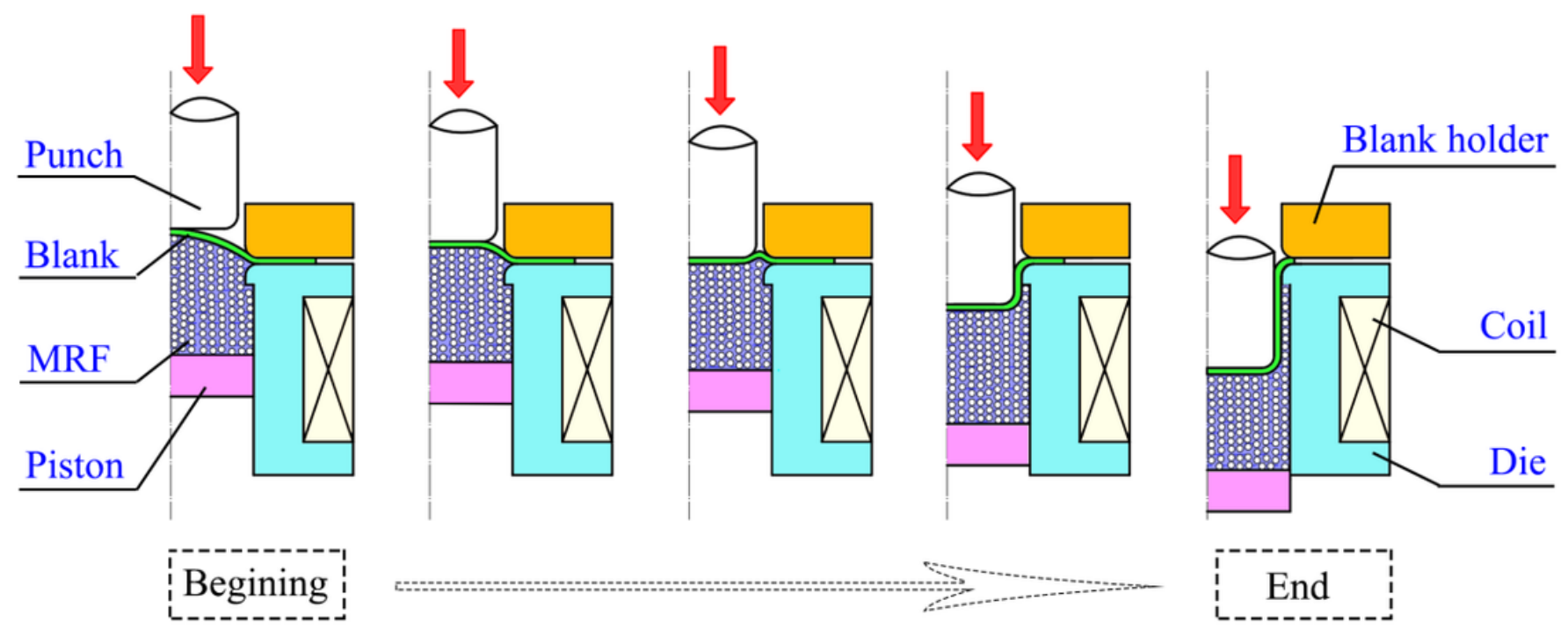

\section{Figure 3}

Schematic diagram of the inverse bulge deep drawing composite forming process 

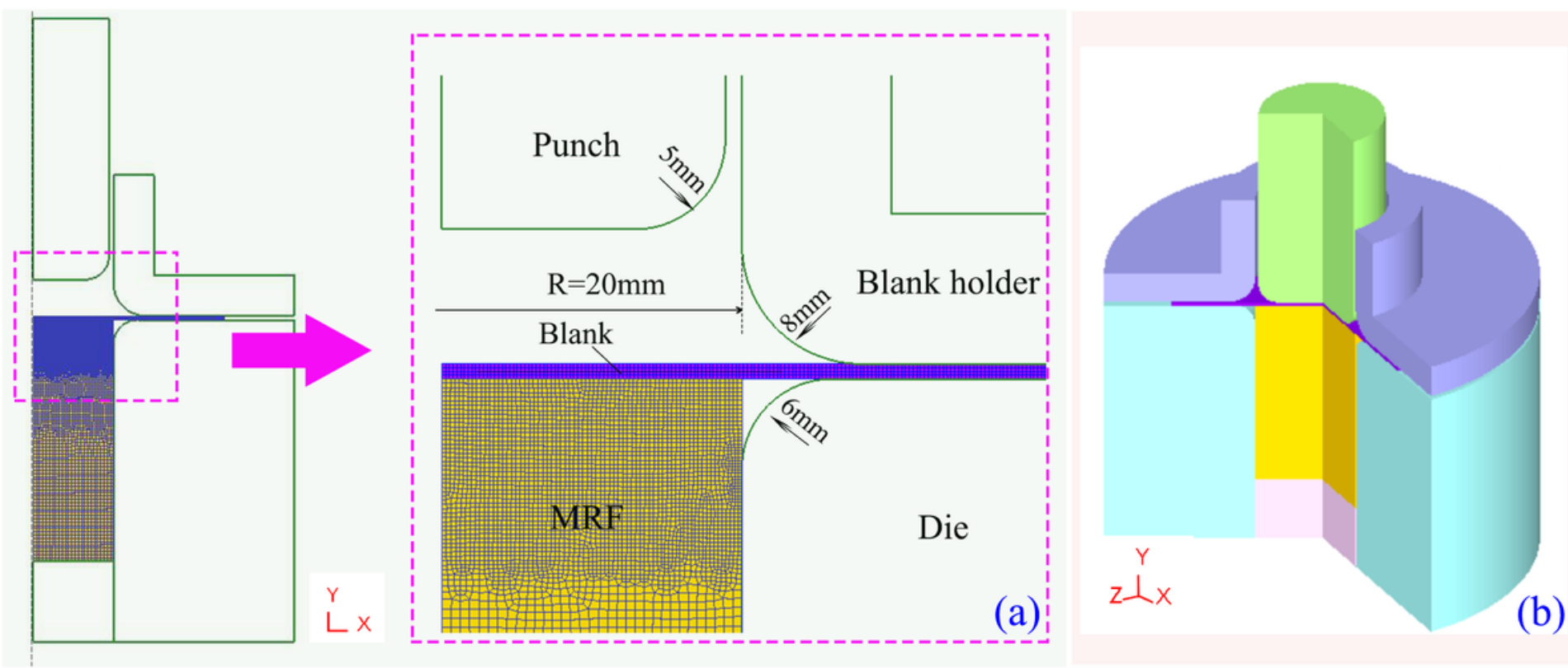

Figure 4

Bending Deformation Model of Single Particle Chain under Compression 


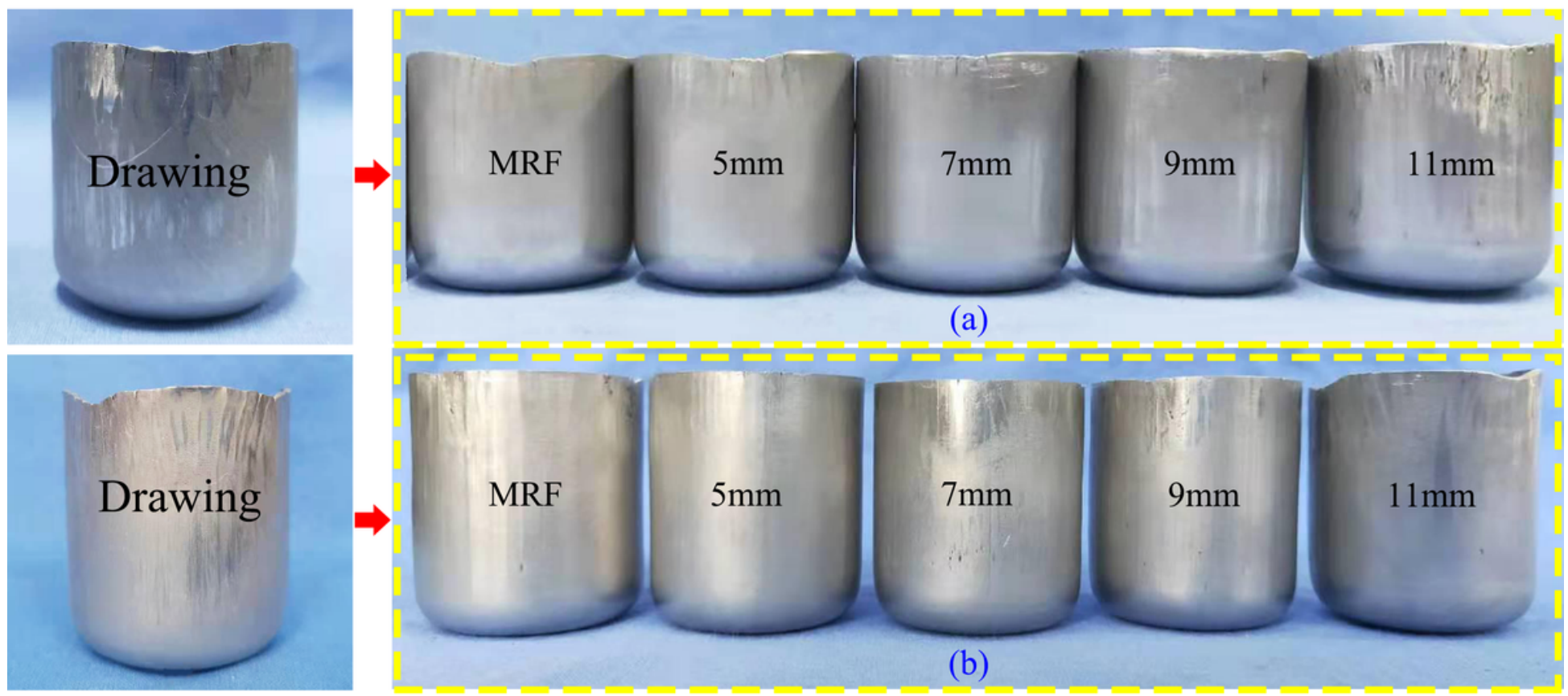

\section{Figure 5}

Cylindrical parts under different forming processes (a) Drawing ratio K1 $=2.125$ forming part (b) Drawing ratio $\mathrm{K} 2=2.25$ forming part 


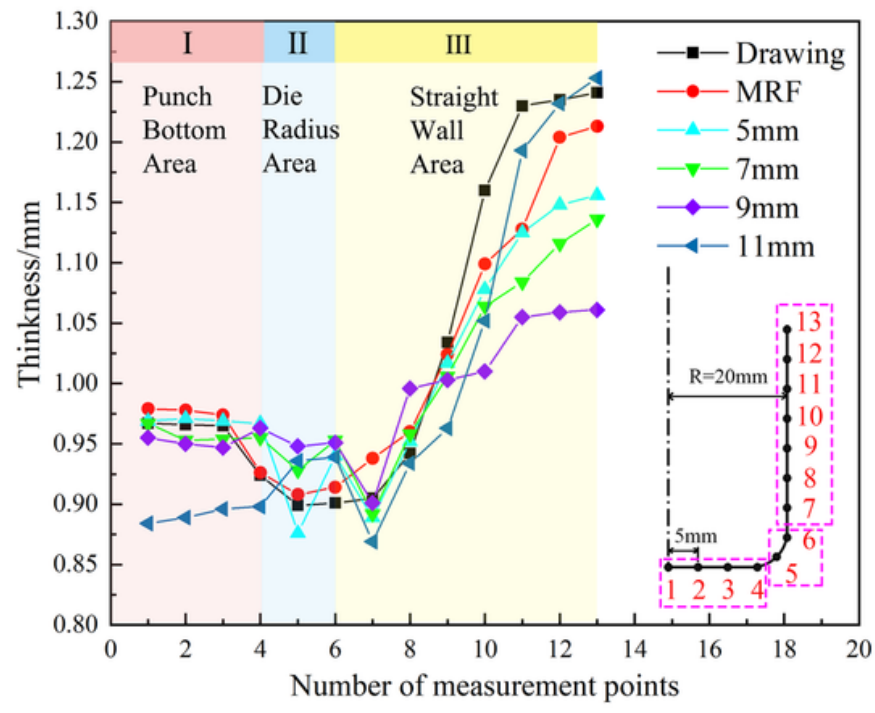

(a)

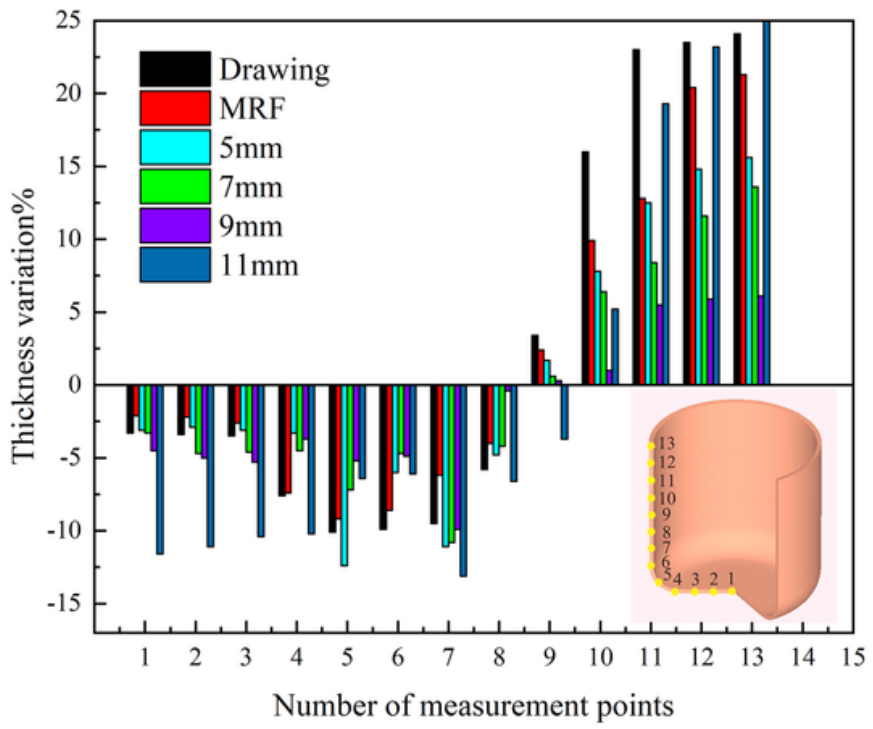

(c)

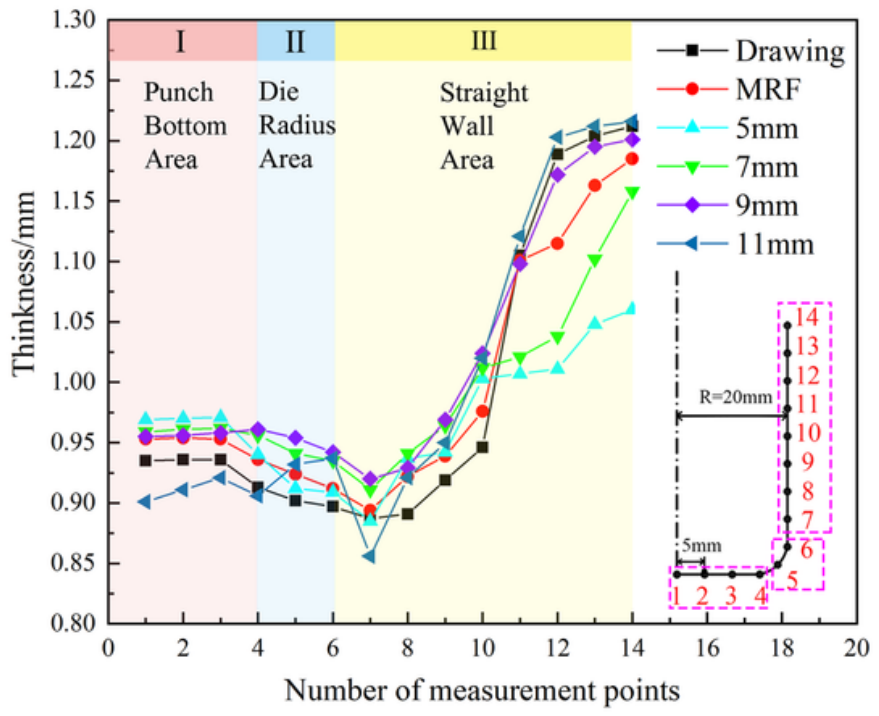

(b)

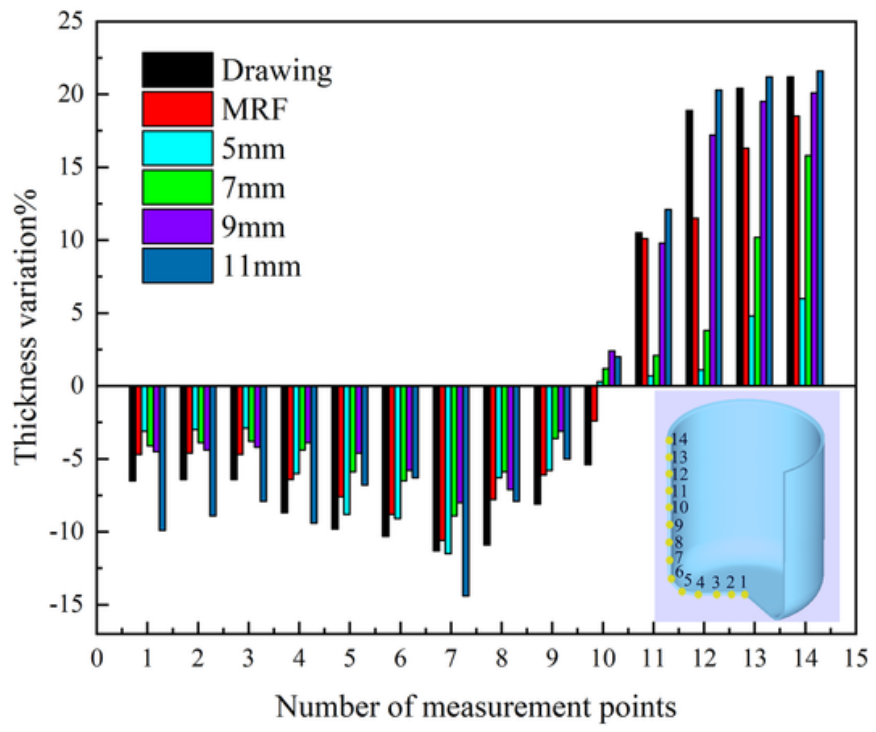

(d)

Figure 6

The distribution of wall thickness of cylindrical parts under different processes (a) $K 1=2.125$, the wall thickness distribution of the formed parts; (b) $\mathrm{K} 2=2.25$, the wall thickness distribution of the formed parts; (c) $\mathrm{K} 1=2.125$, the wall thickness change rate of the formed parts; (d) $\mathrm{K} 2=2.25$, the wall thickness change rate of the formed parts 


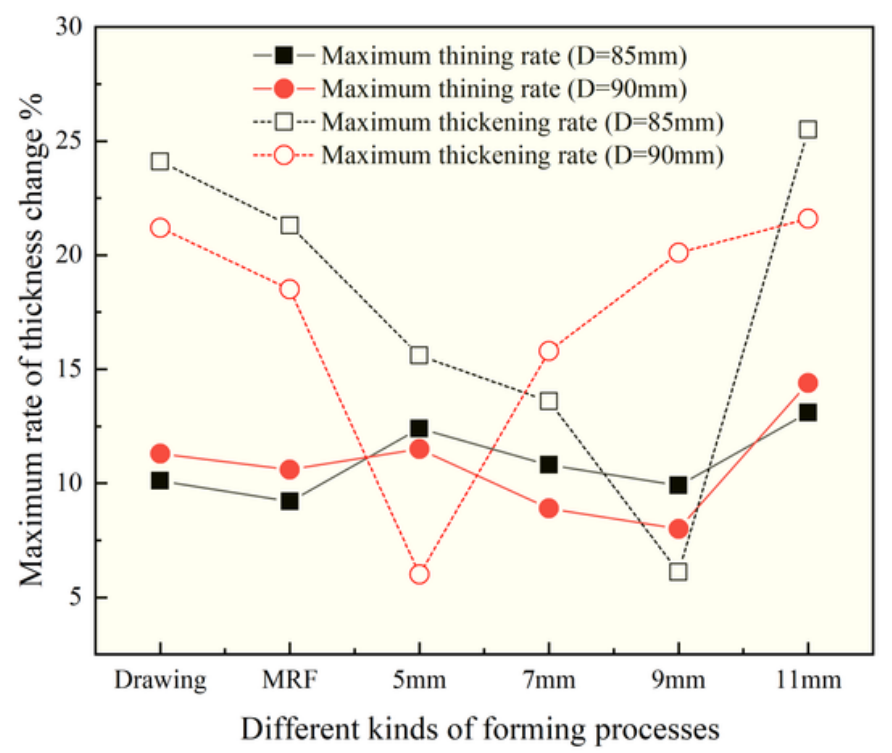

(a)

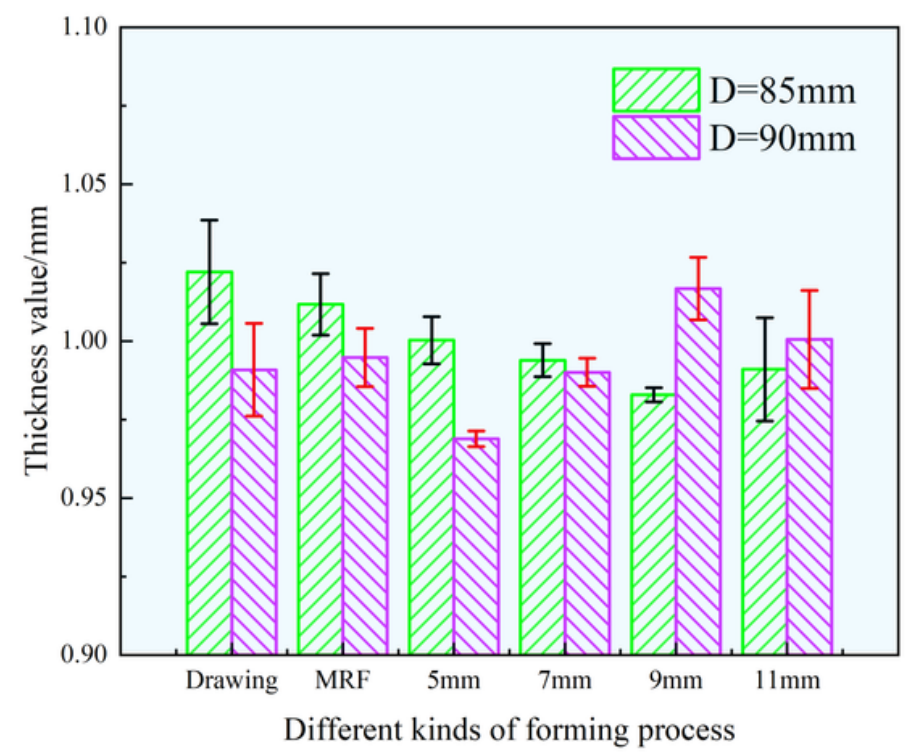

(b)

\section{Figure 7}

Wall thickness changes of cylindrical parts with different forming processes (c) Maximum wall thickness thining rate (d) Mean value and variance of wall thickness distribution 


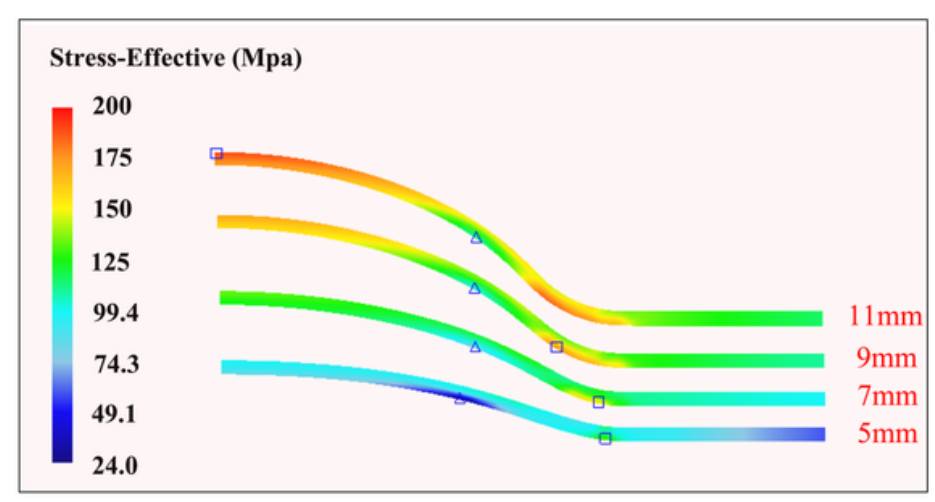

(a)

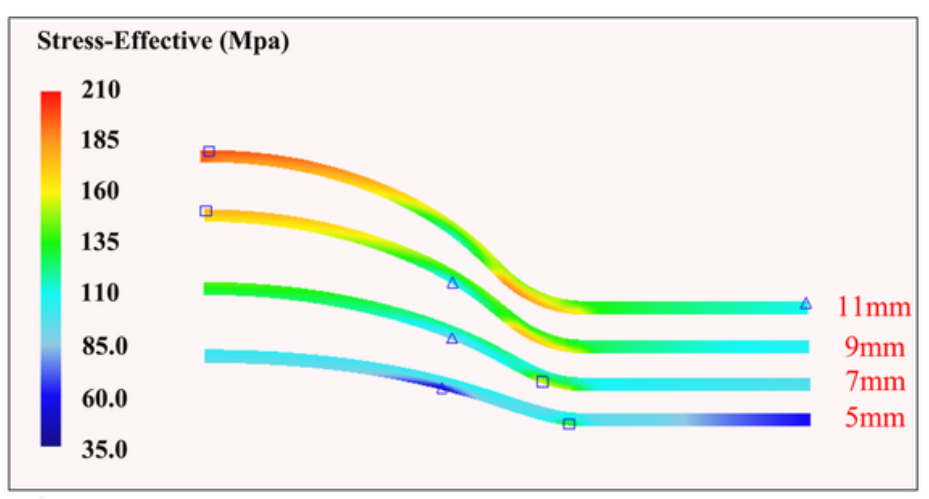

(b)

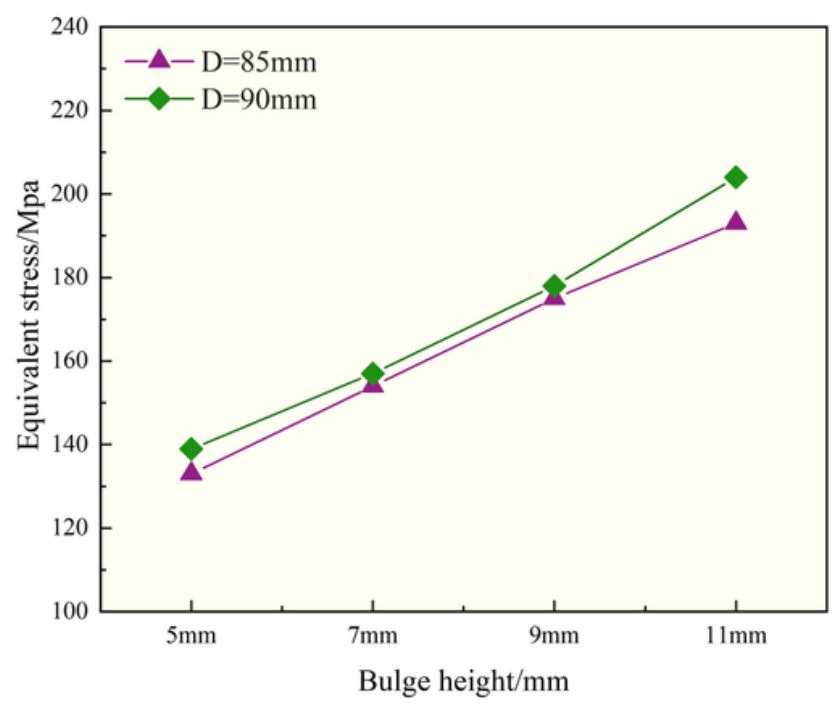

(c)

Figure 8

Equivalent stress distribution of inverse bulge pre-forming of cylindrical parts under different forming conditions (a) Drawing ratio K1=2.125 (b) Drawing ratio K2=2.25 (c) Maximum equivalent stress 


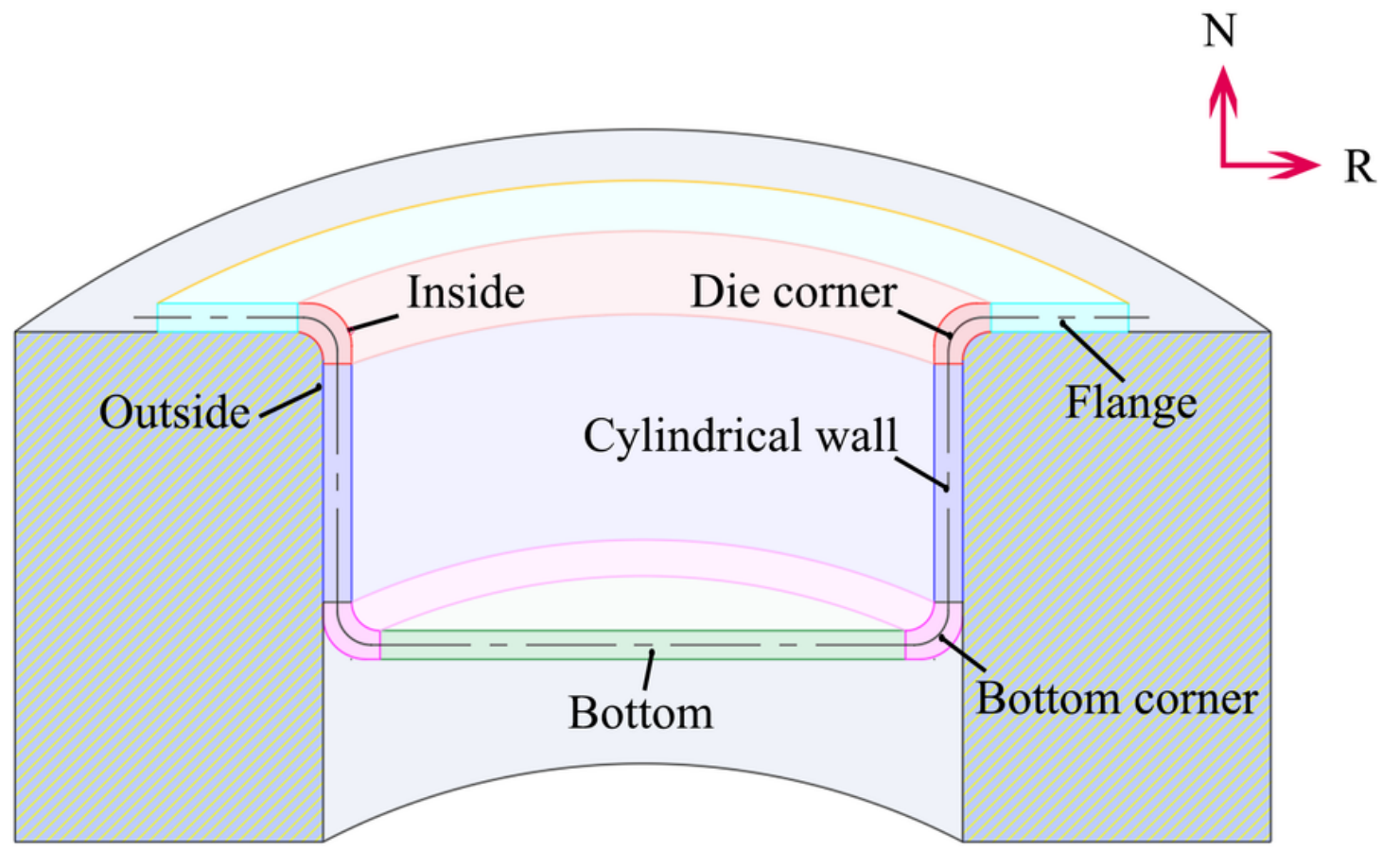

Figure 9

The area of the cylindrical part after deep drawing

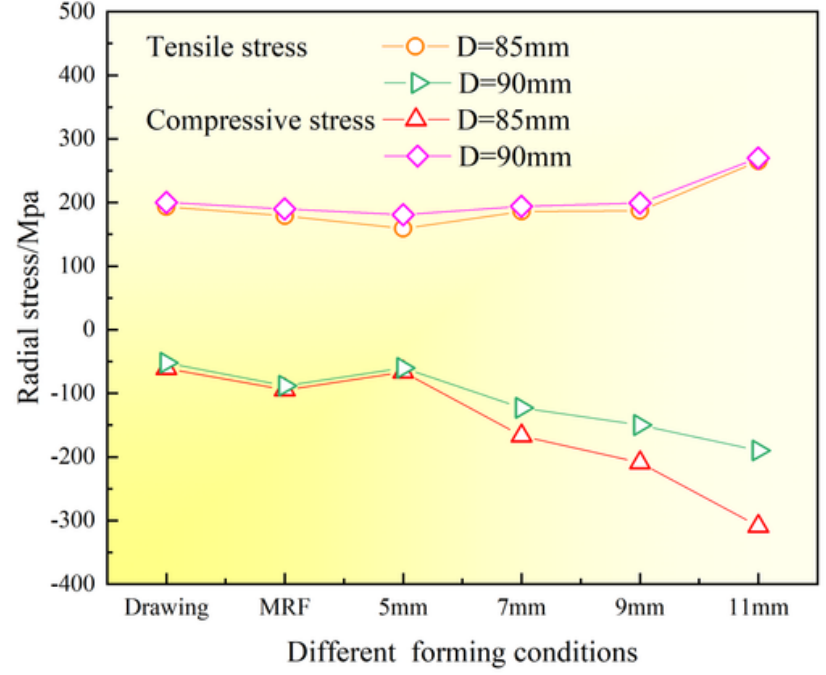

(a)

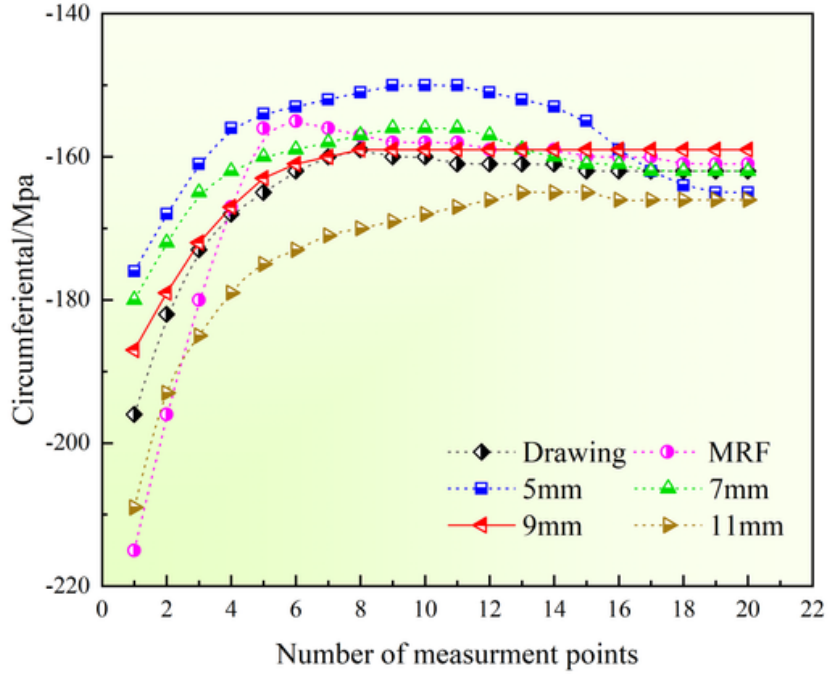

(b)

Figure 10 
The stress of cylindrical parts with different forming processes at the beginning of the deep drawing (a) Radial stress (b) Drawing ratio $\mathrm{K} 1=2.125$ circumferential stress in flange area

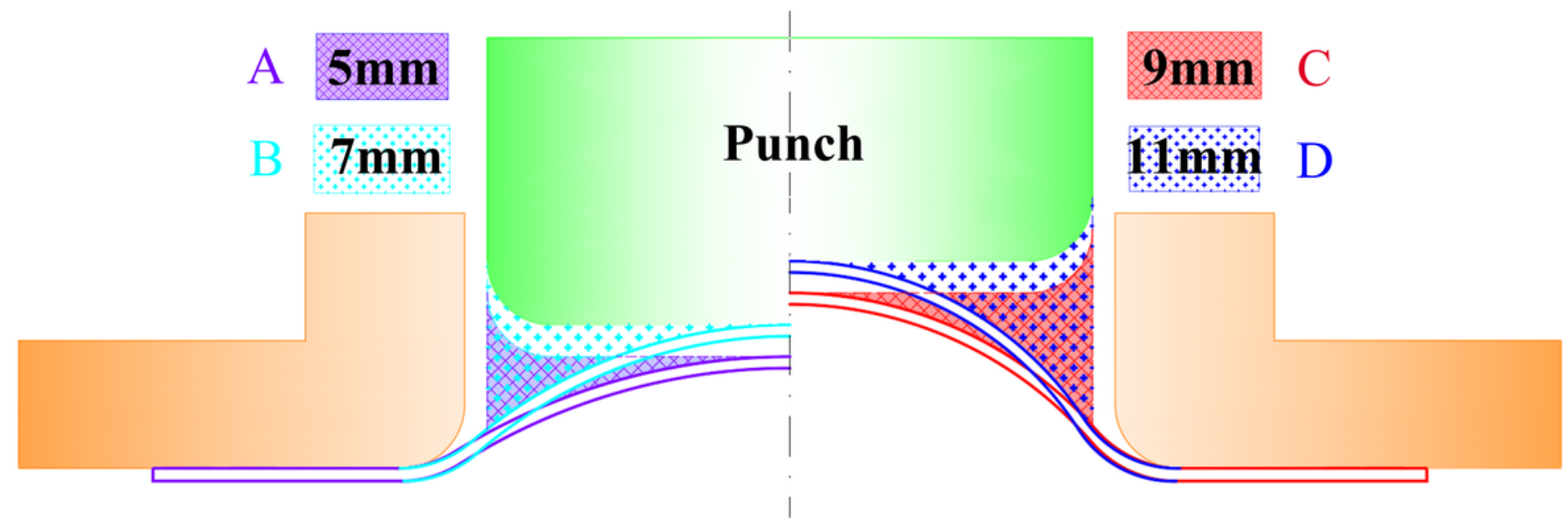

\section{Figure 11}

Suspended area under different pre- forming conditions

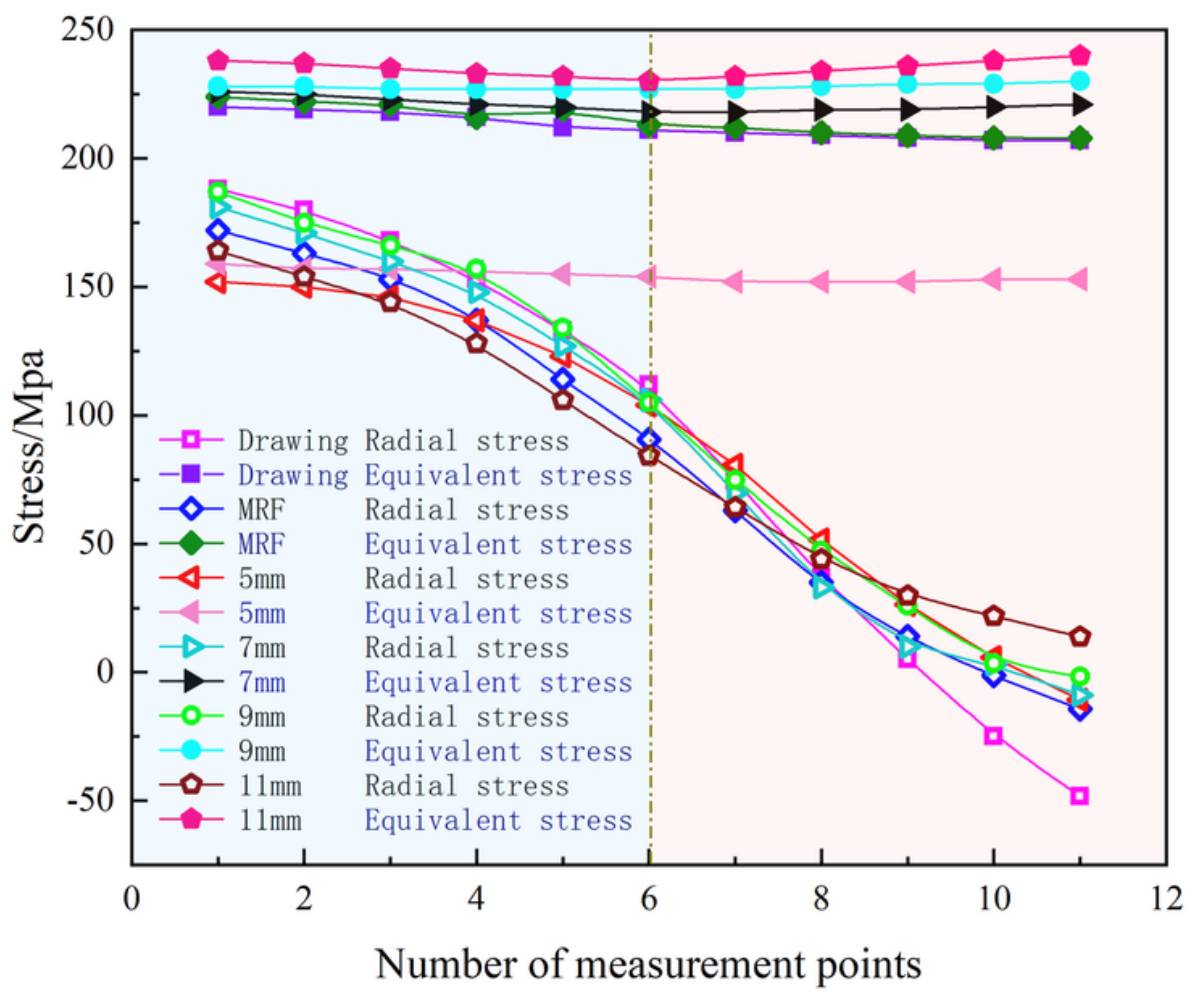


Figure 12

$\mathrm{K} 1=2.125$, the radial stress and equivalent stress distribution along the thickness direction at the corner of the die under different forming conditions 